\section{Using Artificial Neural Networks and Model Predictive Control to Optimize Acoustically Assisted Doxorubicin Release from Polymeric Micelles}

www.tcrt.org

We have been developing a drug delivery system that uses Pluronic P105 micelles to sequester a chemotherapeutic drug - namely, Doxorubicin (Dox) - until it reaches the cancer site. Ultrasound is then applied to release the drug directly to the tumor and in the process minimize the adverse side effects of chemotherapy on non-tumor tissues. Here, we present an artificial neural network (ANN) model that attempts to model the dynamic release of Dox from P105 micelles under different ultrasonic power intensities at two frequencies. The developed ANN model is then utilized to optimize the ultrasound application to achieve a target drug release at the tumor site via an ANN-based model predictive control. The parameters of the controller are then tuned to achieve good reference signal tracking. We were successful in designing and testing a controller capable of adjusting the ultrasound frequency, intensity, and pulse length to sustain constant Dox release.

Key words: Artificial Neural Network; Model Predictive Control; Doxorubicin; Polymeric Micelles; Drug Release; Continuous and Pulsed Ultrasound.

\section{Introduction}

Several therapies are effective in combating abnormal neoplastic tissue growth. One of the methods extensively used to eradicate cancer cells is chemotherapy. Yet chemotherapy has many drawbacks including alopecia, gastrointestinal cramps, leucopenia, and irritation at the site of injection. These side effects are caused primarily because of the non-specific nature of the treatment since systemically delivered drug is able to interact with healthy as well as cancerous cells. Our research group has been developing a drug delivery system that encapsulates the drug until it reaches the tumor site. Then ultrasound is applied to release the chemotherapeutic agent directly to the cancer which would minimize any interaction with the healthy cells in the body. Thus the adverse side effects of chemotherapy are minimized. Our technique uses polymeric micelles to sequester hydrophobic drugs and ultrasound to trigger their release.

Micelles can be formed from simple and small surfactants or from large amphiphilic block copolymers. In applications employing polymeric micelles, hydrophobic drugs are sequestered in the micelle core. Most published applications of US-assisted micellar drug delivery employ polymeric micelles (1-13). The advantages and disadvantages of using copolymeric micelles over other nanosized drug carriers are:

1. They are structurally stable at high concentrations of the copolymer (where they form micelles). Since these molecules have high molecular weights, the
Ghaleb A. Husseini, Ph.D.,2,* Farouq S. Mjalli, Ph.D. ${ }^{3}$ William G. Pitt, Ph.D. ${ }^{2}$ Nabil M. Abdel-Jabbar, Ph.D. ${ }^{1}$

${ }^{1}$ Chemical Engineering Department, American University of Sharjah, Sharjah, United Arab Emirates ${ }^{2}$ Department of Chemical Engineering, Brigham Young University, Provo, Utah

USA 84602

${ }^{3}$ Chemical Engineering Department, University of Malaya, Kuala Lumpur, Malaysia

"Corresponding Author:

Ghaleb A. Husseini

Email: ghusseini@aus.edu 
dissociation time upon dilution is usually longer than other nanoparticles and micelles composed of molecules of lower molecular weights (14).

2. When their corona contains poly (ethylene oxide) (PEO) chains, the micelles are able to circulate in the blood for long times without being recognized and subsequently cleared by the immune system. They are also stable in biological fluids other than blood. In addition, these polymeric compounds do not degrade, and they have long shelf-lives. Recently, a study showed that short chains of PEO tend to adsorb proteins on their surface (15); however PEO chains in Pluronic P105 are sufficiently long that they will prevent such an adsorption from taking place. Pluronic P105 is a block copolymers of poly (ethylene oxide) (PEO) - poly (propylene oxide) (PPO) poly (ethylene oxide) (PEO). It has an average molecular weight of 6500 with the number of monomer units in the PEO and PPO blocks being 37 and 56, respectively. At sufficiently high concentrations, P105 forms micelles, These micelles allow for the sequestration of hydrophobic materials (including drugs) in their core.

3. They are of appropriate size to escape renal excretion $(>15-30 \mathrm{~nm})$ while being large enough to allow for extravasation at a tumor site. With a size this small, a simple filtration process can be used to sterilize the polymer-micelle solution. These micelles can be easily introduced into the circulatory system by intravenous injection $(16,17)$.

4. Hydrophobic drugs can be easily incorporated into copolymer micelles by the simple act of mixing. Such physical entrapment is an efficient and easy way of loading drugs into micellar systems. Physical entrapment has been achieved for several anticancer drugs including Doxorubicin (Dox) and Ruboxyl (Rb) in P105 micelles $(18,19)$.

The main disadvantage associated with micellar carriers is their rapid clearance from the circulation. However by incorporating an exterior layer of PEO, the micelle surface is modified such that they are not cleared as quickly. Because of the hydrophilic nature of PEO, water associates with the poly(ethylene oxide) chains, and this leads to steric repulsion of proteins and a subsequent reduction in protein adsorption on the surface of these drug delivery vehicles. By reducing, and in some cases, preventing protein adsorption, micellar drug carriers remain longer in the blood circulation because they are protected from detection and clearance by several biological mechanisms (20). While these carriers are important in our drug delivery system, the release or trigger mechanism (ultrasound) is equally important.

Ultrasound (US) has been investigated by several groups as a potential facilitator of the delivery and absorption of drugs (21-23). Early studies on transdermal drug delivery using higher frequencies available in diagnostic equipment had limited success (24-26), but Mitragotri using lower ultrasonic frequencies $(20 \mathrm{kHz})$ achieved transdermal delivery of medium molecular weight proteins (insulin, interferon, and erythropoeitin) (27). His hypothesis was that cavitation events disrupted the stratum corneum. In our drug delivery work we believe that cavitation disrupts micelles, leading to drug release. Kruskal et al. have reported that higher frequency ultrasound (imaging frequencies) increased the permeability of blood vessels and increased the quantity of Dox delivered by stable liposomes to hepatic colorectal metastases in a mouse model (28). Thus US may further increase the enhanced permeability of tumor capillaries which already enable some passive targeting of tumors by nanosized drug carriers $(29,30)$. Kwok et al. demonstrated ultrasonic-activated release of insulin from a monolithic drug reservoir with an impermeable surface coating that is disrupted by the action of ultrasound (31). After insonation is stopped, the coating reforms and blocks further release of the drug. In addition to triggering the release, ultrasound is credited with causing or enhancing chemical reactions that can be chemotherapeutic (32-34).

We have been focusing much of our attention on modeling acoustically activated drug release and re-encapsulation from polymeric micelles $(2,10,11)$. Modeling such a system is interesting because of the ease with which the release can be controlled, simply by increasing or decreasing the length and the intensity of the acoustic pulse. In a previous publication, we reported on the use of Artificial Neural Network (ANN) algorithms to deduce the sensitivity of acoustically activated drug release from polymeric micelles (10), because we found that conventional linear modeling techniques are incapable of capturing the transient nature of this highly nonlinear process. Black-box modeling algorithms are capable of modeling nonlinear systems and are gaining the scientific community acceptance because of their simplicity and high prediction performance (35). ANN modeling techniques are attractive for use in drug delivery because no prior knowledge of the process mechanism (usually needed to generate the pharmacokinetic models) is required. To model any system using ANN, all one needs is the input-output data from the process being investigated. This data will be used first to train the neurons and later to validate and test the resulting model.

In addition to modeling, Neural Networks are currently being used to devise controllers in a variety of fields. They are either directly implemented where the network controller is trained to learn the inverse of the process dynamics, or indirectly implemented by training the neural network to predict future outputs from past and present inputs and outputs. In the former case, the process is modeled with a separate neural network; the controller does not invert the 
exact process model and the offset cannot be eliminated. The indirect method is more suitable for control applications. The trained process model is used with a control algorithm to calculate the controller output. ANN-based control algorithms are implemented in Model Predictive Control (MPC) (36, 37), Internal Model Control (IMC) (38), Dynamic Matrix Control (DMC) (39), and Adaptive Control (40). The application of ANN nonlinear control design techniques in modeling drug delivery (namely, release and re-encapsulation of Doxorubicin from Pluronic P105 micelles) systems is unique as well as promising. In this article, we extend our previous ANN dynamic modeling efforts (10) to propose an neural networks-based MPC controller capable of maintaining a steady state profile of drug release from micelles using ultrasound. ANN is particularly useful to model this system because of the strong nonlinearity and high noise aspects of the experimental data available. As with all neural network/control based algorithms, the validated model is then utilized to train an ANN-based model predictive controller. Finally, the controller parameters are tuned to achieve acceptable reference signal tracking. The proposed controller is capable of adjusting the ultrasound frequency, intensity, and the pulse length to sustain a constant release of Doxorubicin from Pluronic P105 micelles. Next we will discuss the ANN based control model/algorithm.

\section{Model Predictive Control}

Model based predictive control (MPC) controllers are designed to drive the process from one constrained steady state to another. They may receive an optimal steady-state operating point from an overlying optimizer, or they may compute an optimal operating point using an internal steadystate optimizer. MPC was successfully applied in solving process control problems (41). This is basically related to its ability to deal with complex situations such as systems with large delays, process variables constraints, non-minimum phase systems. Another success is its robust performance against model inaccuracies (42). The basic implementation of MPC was mainly for linear systems. Recently, great effort is being devoted for the development and implementation of nonlinear versions of this algorithm. One of these implementations is the use of neural networks for controller design purposes. Neural networks are capable of capturing the system nonlinear dynamics and can be used to approximate the process as well as to design the model predictive controller. The neural network based model predictive controller (NNMPC) uses a neural network model of a nonlinear plant to predict future plant or process performance. The controller then calculates the predicted control input that will optimize the plant or process performance over a specified future time horizon. This control method is based on the receding horizon technique (43).
The NN-MPC structure used in this study is shown in Figure 1. It is composed of four components in addition to the process. These components are; two neural networks (one for the process and the other for the controller), an optimizer and a performance function. For a selected time horizon, the controller optimizes the process output by using the neural network model for calculating controller moves and predicting the plant or process output. The neural network controller is trained using real process input-output data in order to produce the correct controller moves generated by the optimization algorithm.

Basically, the NN-MPC solves for the control signal variable $\boldsymbol{u}^{\prime}$ by minimizing the following objective function:

$$
\begin{gathered}
J(t, \mathrm{U})=\sum_{i=N_{1}}^{N_{2}}\left[y_{r}(t+i)-y_{m}(t+i)\right]^{2}+ \\
\lambda \sum_{i=1}^{N_{u}}\left[u^{\prime}(t+i+1)-u^{\prime}(t+i-2)\right]^{2}
\end{gathered}
$$

Where, $\lambda$ is the control weighting parameter that determines the contribution of the sum of the squares of the control prediction increments have on the performance index. The $N_{l}$ and $N_{2}$ are the minimum and the maximum prediction horizons. $N_{u}$ is the "controller's move horizon." It specifies the instant time, when the output of the controller should be kept at a constant value. The $\mathbf{U}$ is the $N_{u}$ future control moves vector defined as: $\mathrm{U}=\left[u(t), u(t+1), \ldots, u\left(t+N_{u}-1\right)\right]^{T}$. Nørgaard et al. (44) reported the derivation of the controller in details.

The controller parameters can be used to tune the performance of the predicted output. This usually requires some exploratory experiments for determining the best controller parameters. Depending on the problem formulation, the main tuning parameters may involve one or more of the following; sampling time, control horizon, prediction horizon, and weighting matrices in the optimization formulation $(45,46)$.

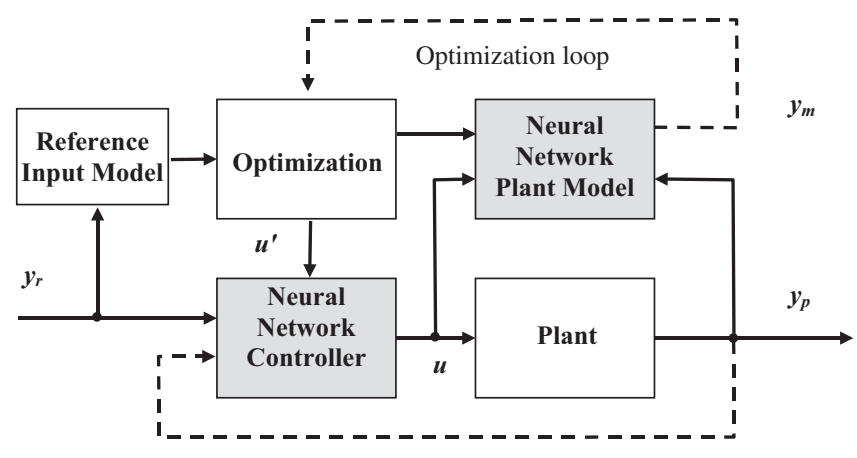

Figure 1: The neural network based MPC structure. 


\section{Materials and Methods}

\section{Pluronic P105}

Pluronic P105 was found to be an ideal drug carrier for ultrasonic-activated drug release for several reasons. First, it forms micelles quickly upon simple dissolution in water (16). Second, the core of PPO is sufficiently hydrophobic to stabilize the micelle and sequester hydrophobic drugs (47). Third, the micelles can be perturbed by low frequency ultrasound to release the drug (3). The drug is quickly re-encapsulated in the carrier when insonation is stopped $(3,7,8)$. Finally, Pluronic compounds at low concentrations are non-toxic and can be cleared by the kidneys (48).

We briefly experimented with other Pluronic (triblock copolymer of the form $\mathrm{PEO}_{\mathrm{x}}-\mathrm{PPO}_{\mathrm{y}}-\mathrm{PEO}_{\mathrm{x}}$ ) compounds, but found them unsatisfactory when used as pure Pluronics because those with longer PEO blocks had too high of a critical micelle concentration (CMC), and those with longer PPO blocks could not dissolve easily in water. Thus the composition of Pluronic P105 appears to be close to optimal for drug sequestration and ultrasonic release.

Pluronic P105 was purchased from BASF Corporation. The surfactant was dissolved in a phosphate buffered saline (PBS) solution. The final concentration of the Pluronic solutions used in our study was $10 \mathrm{wt} \%$. These solutions were sterilized by filtration through a $0.2 \mu \mathrm{m}$ filter.

\section{Doxorubicin}

Doxorubicin (Dox) was purchased from Sigma and from the University Hospital (University of Utah, Salt Lake City, UT) and was dissolved in a stock solution of phosphate buffered saline (PBS). This drug is presently being used extensively in the therapy of several cancers. Dox received from Sigma was $98 \%$ Dox and $2 \% \mathrm{HCl}$, while Dox purchased from the University Hospital was one sixth Dox and the balance lactose. Anthracycline drugs (including Dox) are topoisomerase I and II inhibitors. These compounds intercalate with DNA, affecting many of its functions, including the synthesis of DNA and RNA. These drugs cause single- and double-strand breaks to occur in the DNA, which leads subsequently to cell death (49). Dox was introduced into PBS or Pluronic P105 micellar solutions from a stock solution in PBS $(1 \mathrm{mg} / \mathrm{ml})$. In our release experiments, a drug concentration of $6.67 \mu \mathrm{g} / \mathrm{ml}$ was used.

\section{Ultrasound}

Ultrasound power at $70 \mathrm{kHz}$ was generated by a Sonicor SC-100 ultrasonicating bath (Sonicor Instr., Copaique, N. Y.). The power density (acoustic intensity) was controlled by adjusting the input voltage using a variable A.C. transformer
(Variac). The insonation intensity as a function of applied voltage was determined using a calibrated hydrophone (Bruel and Kjaer model 8103, Decatur, GA). The acoustic intensity generated by the Sonicor bath increased with applied voltage and the intensity dropped below detection level when less than $60 \mathrm{~V}$ A.C. were applied from the Variac.

Twenty-kHz ultrasound was generated by a probe transducer (Sonics and Materials, Newton, CT) inserted into the water bath. The $20-\mathrm{kHz}$ ultrasound probe was programmed to generate continuous wave $(\mathrm{CW})$ or pulsed ultrasound of varying power densities and duty cycles.

\section{Measuring Drug Release}

We have developed a laser fluorescence detection system to quantify the amount and the kinetics of Dox release from these micelles $(3,7)$. The system consists of an argon-ion laser at 488 $\mathrm{nm}$ directed into a glass cuvette containing the trial solution to be insonated. A fiber optic probe is used to collect the fluorescence emission from the cuvette. The collected light passes through a bandpass filter centered at $535 \mathrm{~nm}$ to a sensitive silicon detector, whose signal is digitized and stored on a computer. The temperature of the ultrasonic exposure chamber is maintained at $37^{\circ} \mathrm{C}$. A decrease in fluorescence is attributed to release of the Dox from the micelle core to the aqueous phase where its fluorescence is partially quenched by water, and the release was quantified using a calibration with free Dox (3). The percent release was calculated using the following:

$$
\% \text { Release }=\frac{I_{P 105}-I_{U S}}{I_{P 105}-I_{P B S}} \times 100 \%
$$

and, the encapsulation fraction, the fraction of Dox inside the micelle, as:

$$
\% \text { Encapsulation }=\left(1-\frac{I_{P 105}-I_{U S}}{I_{P 105}-I_{P B S}} \times 100 \%\right)
$$

where $I_{P 105}, I_{U S}$, and $I_{P B S}$ refer to the fluorescent intensities of Dox in P105 solution (no US), Dox in P105 with application of US, and Dox in a PBS solution, respectively.

Results using this system revealed that up to $10 \%$ of the Dox is released, depending upon the insonation intensity (3). Pulsed insonation resulted in pulsed drug release and re-encapsulation (2). Analysis of the kinetics of the release and re-encapsulation showed that Dox release was zero order with respect to Dox concentration (but a strong function of insonation parameters) and re-encapsulation was first order in free Dox concentration $(2,11)$. Both release and re-encapsulation were completed within 0.6 seconds at any frequency. Energy deposition does not appear to be a requirement to release drugs. The release appears to occur at 
isothermal conditions, and in water that has a very low attenuation, particularly at these low frequencies. Thus exposure to the pressure field, and not energy deposition, releases the drug from the carrier.

\section{Results and Discussion}

Figures 2 and 3 show the two sequences of experimental data points collected at 20 and $70 \mathrm{kHz}$, respectively. For both sequences, the three profiles of the three pulses indicate a nonlinear behavior for the drug release process. The three power densities shown in Figure 2 at $20 \mathrm{kHz}$ are $0.058,0.047$ and $0.033 \mathrm{~W} / \mathrm{cm}^{2}$. These intensities produced new steady state encapsulated fraction averages of $0.9150,0.9203$ and 0.9613 , respectively. Similarly for the case of $70 \mathrm{kHz}$ (see Figure 3), the three acoustic power densities used $(0.765,0.675$ and 0.58 $\mathrm{W} / \mathrm{cm}^{2}$ ) resulted in the new steady state encapsulated fraction averages of $0.9049,0.9112$ and 0.9348 , respectively. It is clear that the three ultrasound intensities, at both frequencies, achieved steady state encapsulated drug fraction levels that are not linearly proportional to the power densities of the ultrasound introduced. This nonlinearity necessitates the need to use a non-conventional control technique that is capable of handling such process complexities accurately and efficiently.

\section{Modeling and Controller Design Results}

As mentioned in the introduction section, a nonlinear model based control strategy should be capable of attaining good reference tracking with minimum controller moves for complex
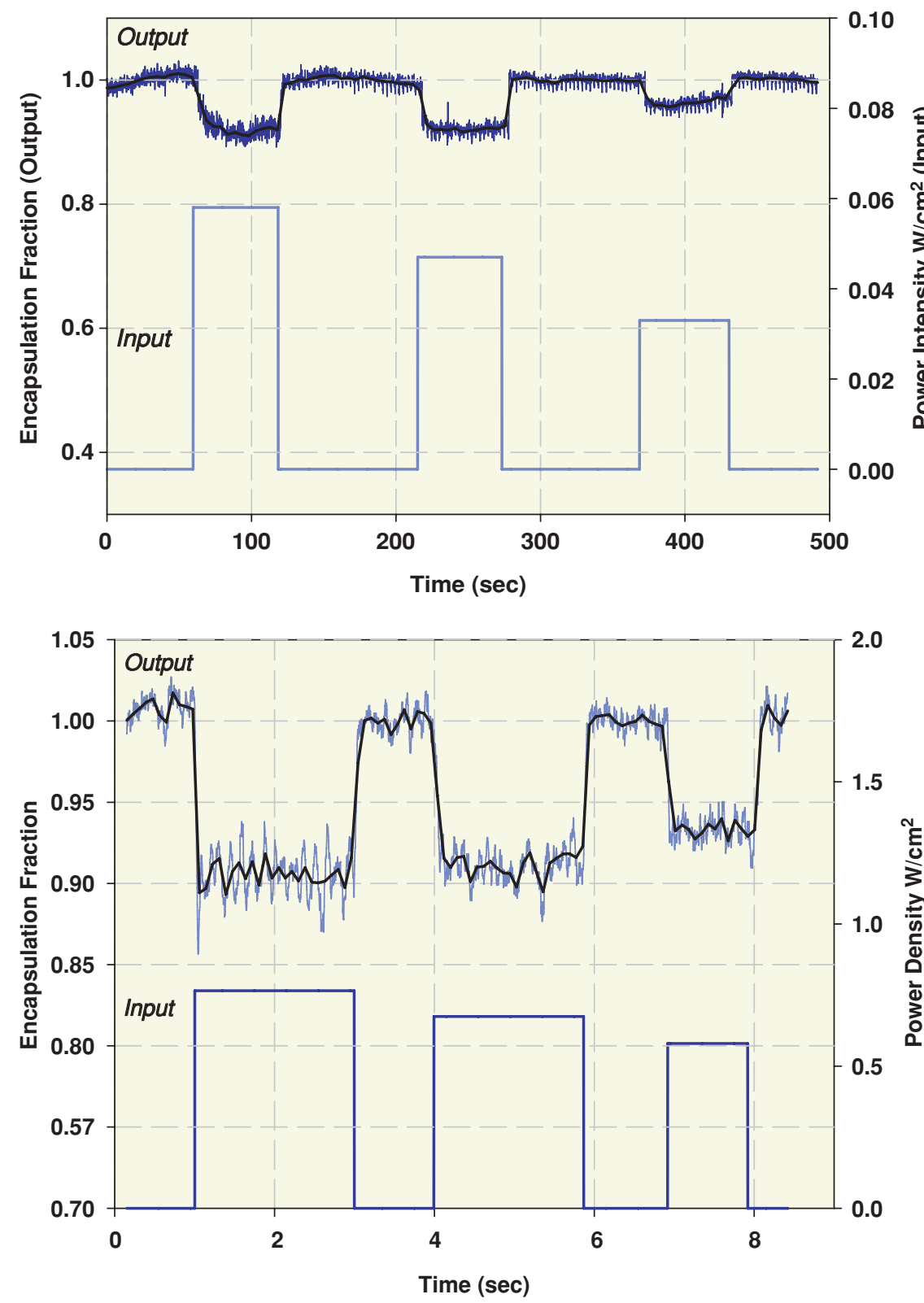

Figure 2: Raw and smoothed experimentally generated drug release data and the corresponding power density signals (at $20 \mathrm{kHz}$ ).
Figure 3: Raw and smoothed experimentally generated drug release data and the corresponding power density signals (at $70 \mathrm{kHz}$ ). 
processes such as the drug delivery process at hand. Adopting such a control algorithm necessitates the computation of a neural network equivalent dynamic model of the drug release process. The input-output data were first smoothed to get rid of measurement noise and prepare it for ANN training. A moving average smoothing strategy was used with a sampling proportion of 0.01 . The smoothed data (see Figures 2 and 3 ) revealed the nonlinearity, especially within the periods where the power density was changed.

A feedforward ANN with output feedback was constructed with one hidden layer. The general structure of this network is shown in Figure 4. The neural networks structure was selected based on testing different network topologies that vary in terms of structure and simulation parameters. The criteria for network structure selection are based on its simplicity, performance, and accuracy of model prediction. The finally selected network consists of a single hidden layer with 3 neurons. The activation function used in the hidden layer is the hyperbolic tangent sigmoid transfer function while the output layer contains a linear neuron. Two delayed process outputs were used in the network to account for the time variation of the variables in the system. The inputs and targets data were preprocessed by normalization so that they fall in the interval $[-1,1]$. This makes the neural network training more efficient. Network training was accomplished by manipulating its weights and biases to achieve certain performance criteria. This was accomplished using an optimization algorithm that searches for network parameters capable of minimizing the prediction error $\left(\bar{\varepsilon}_{s}\right)$ described by the mean square errors between the experimental encapsulation fraction, $y_{i}$, and the network predictions $\hat{\mathrm{y}}$ for $n$ sampling points expressed as:

$$
\bar{\varepsilon}_{s}=\frac{1}{n} \sum_{i=1}^{n}\left(\hat{y}_{i}-y_{i}\right)^{2}
$$

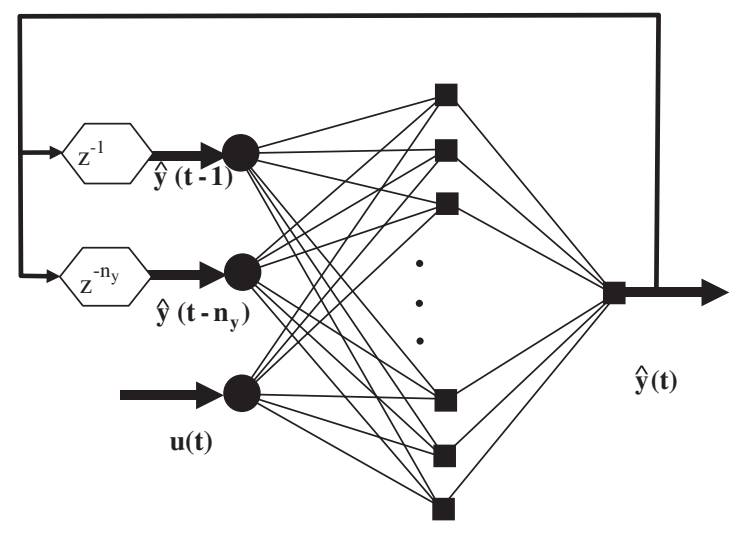

Input layer Hidden layer Output layer

Figure 4: Structure of a feed foreword neural network with time delayed feed.
The MATLAB software implementation of the LevenbergMarquardt back-propagation optimization algorithm (LMBP) was used for this purpose. The algorithm converges to the optimal solution with minimum optimization steps.

The $20-\mathrm{kHz}$ and $70-\mathrm{kHz}$ input/output data sets pairs were then divided into three subsets with the ratio of 2:1:1 (training, validation and testing, respectively). At $20-\mathrm{kHz}$, a total of 514 epochs (training steps) were needed to achieve prediction errors of $1.07 \times 10^{-6}, 2.10 \times 10^{-6}$ and $7.1 \times 10^{-6}$ for the three respective subsets within a search time of 104 seconds. On the other hand, the $70-\mathrm{kHz}$ data needed 431 epochs to attain the training target and achieve prediction errors of $5.1 \times 10^{-6}$, $8.12 \times 10^{-6}$ and $4.2 \times 10^{-6}$ for the three respective subsets within a search time of 93 seconds. With this performance, the trained ANN can predict process dynamics accurately. Figure 5 shows the profiles of prediction errors for the training, validation and the testing subsets for the $20 \mathrm{kHz}$ data, while Figure 6 depicts the same information for the $70 \mathrm{kHz}$ data. The ANN predictions are very close to the actual drug release data with

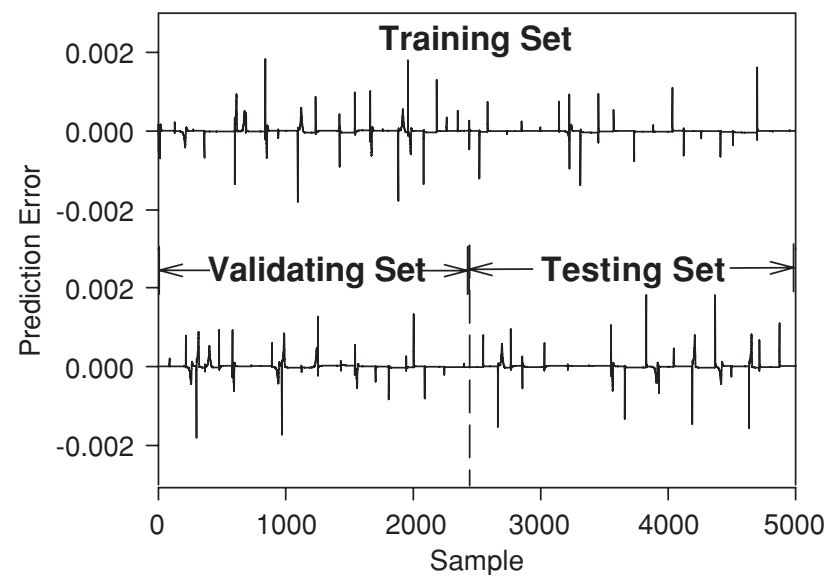

Figure 5: ANN Prediction Performance for the three data sets at $20 \mathrm{kHz}$.

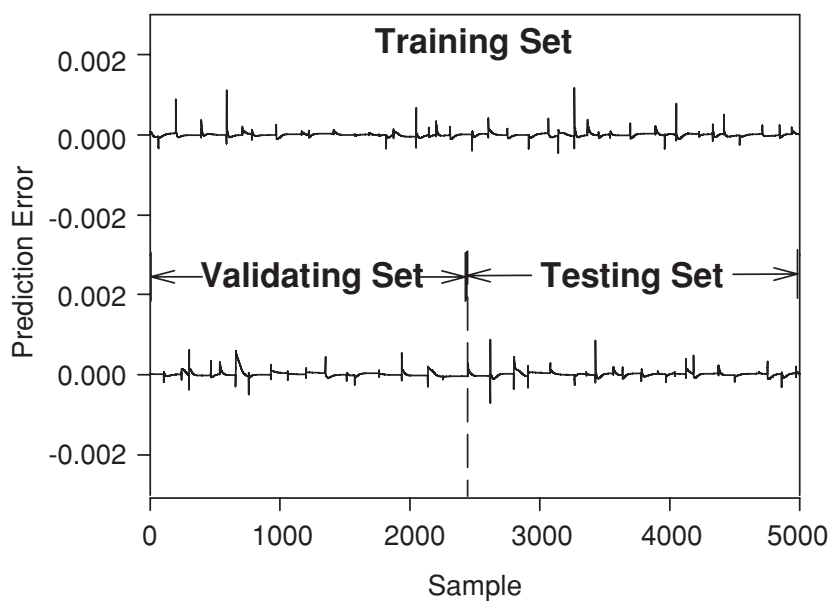

Figure 6: ANN Prediction Performance for the three data sets at $70 \mathrm{kHz}$. 


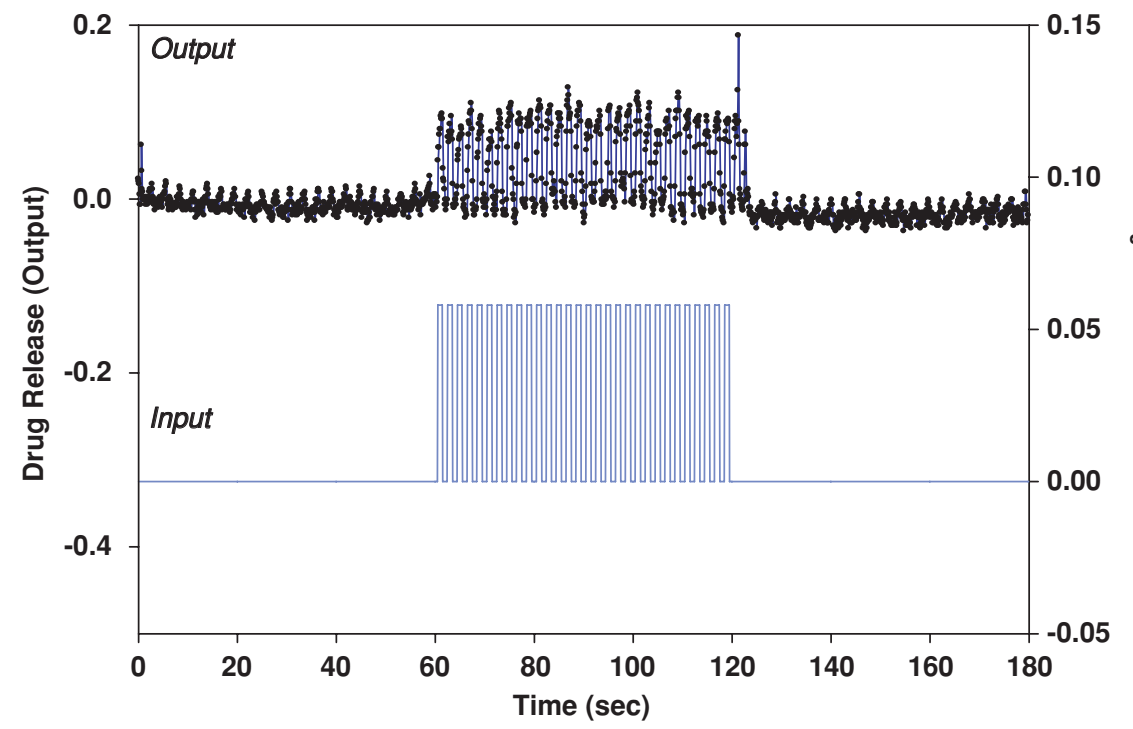

Figure 7: Experimental data and Model output overlaid (top) for an input of 1 Second "On": 1 second "OFF" pulsed ultrasound at $20 \mathrm{kHz}$ and $0.058 \mathrm{~W} / \mathrm{cm}^{2}$ (bottom). The model accurately captures the kinetic behavior of release and re-encapsulation of pulsed ultrasound.

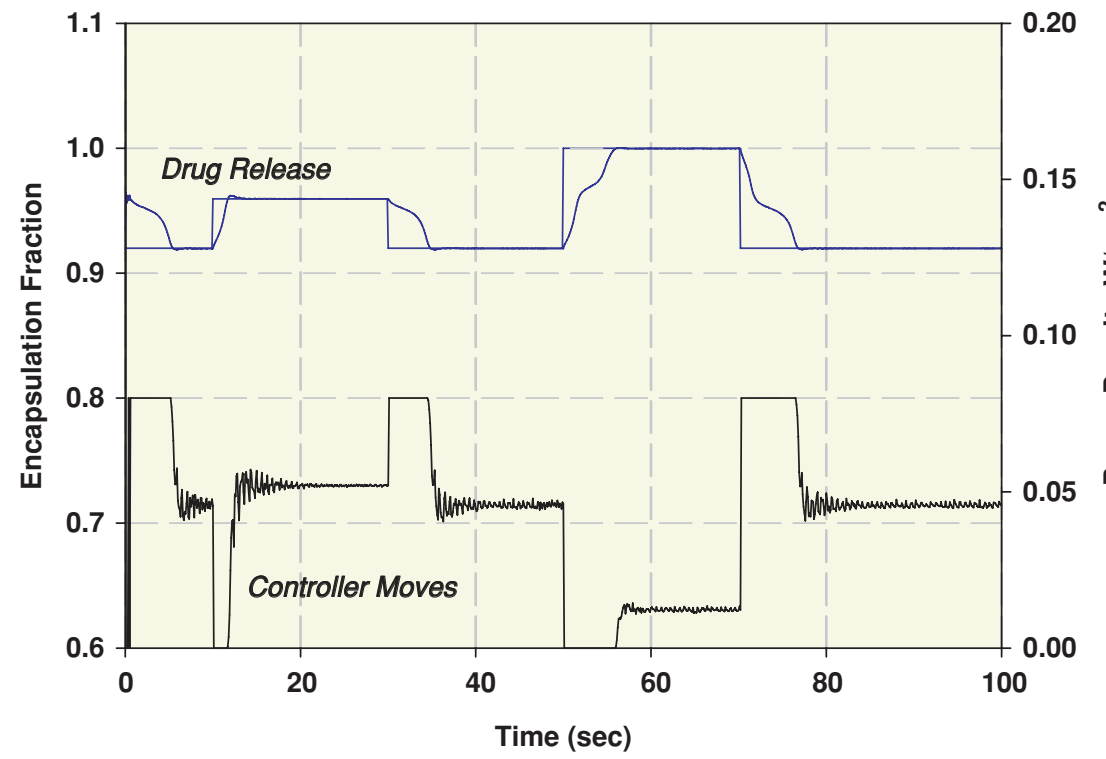

Figure 8: NN-MPC setpoint tracking performance and the corresponding controller (at $20 \mathrm{kHz}$ ).

a maximum prediction error of 0.002 and 0.001 at $20 \mathrm{kHz}$ and $70 \mathrm{kHz}$, respectively. Figure 7 shows a comparison between experimental data and model output for an input of 1 second "On": 1 second "OFF" pulsed ultrasound at $20 \mathrm{kHz}$ and 0.058 $\mathrm{W} / \mathrm{cm}^{2}$. The figure clearly shows that the model accurately captures the kinetic behavior of release and re-encapsulation even when the duration of the ultrasound pulse is 1 second or less.

After modeling the drug release process dynamics, the generated trained ANN was implemented in the NN-MPC controller. The controller structure of the model is based on feedforward neural networks. The structure of the controller is given by Liu et al. (50). The design of the controller F involves tuning its parameters. From the controller objective function given in equation [1], the tuning parameters for this controller are: the prediction horizon $\left(N_{2}\right)$, the control horizon $\left(N_{u}\right)$, the control weighting factor $(\lambda)$ and the search parameter $(\alpha)$. These parameters were selected as described next. The prediction and control horizons were set at their best values of 10 and 5, respectively, after attempting other values. These values showed moderate aggressiveness and a good stability of the controller response. The NN-MPC control weighting factor was set at $(\lambda=0.1)$. This value resulted in a smooth process output and gave good tracking with low oscillatory behavior.

The last tuning parameter is the search parameter $(\alpha)$. This parameter is used to control the optimization speed and performance. It determines the termination of the search process. The Levenberg-Marquardt optimization algorithm uses this scale factor to minimize the performance training function along the search direction. After several trials, a value of 0.001 was selected to insure reasonable performance criteria and optimization convergence speed.

To test the final design of the NN-MPC controller, a series of set-point excitations in the drug release (or drug encapsulation fraction) were introduced in the process control loop during which the controller performance was recorded. The resulting profile of the process and the corresponding controller moves for the $20-\mathrm{kHz}$ and $70-\mathrm{kHz}$ cases are shown in Figures 8 and 9, respectively. In terms of controller moves, the NN-MPC configuration produced smooth and non-aggressive changes in the power density. The controller output was bounded within the allowable limits of ultrasound intensity range $\left(0-0.058 \mathrm{~W} / \mathrm{cm}^{2}\right.$ and $0-0.756 \mathrm{~W} / \mathrm{cm}^{2}$ for the two respective frequencies). The figures indicate that the controller was able to direct the process to track the reference signals excitations safely, smoothly and within a reasonable response time. Figure 10 shows the response of the controller as the percent drug release is increased to $5 \%$ (hence, the fraction of drug encapsulated decreased from 1 , or $100 \%$, to 

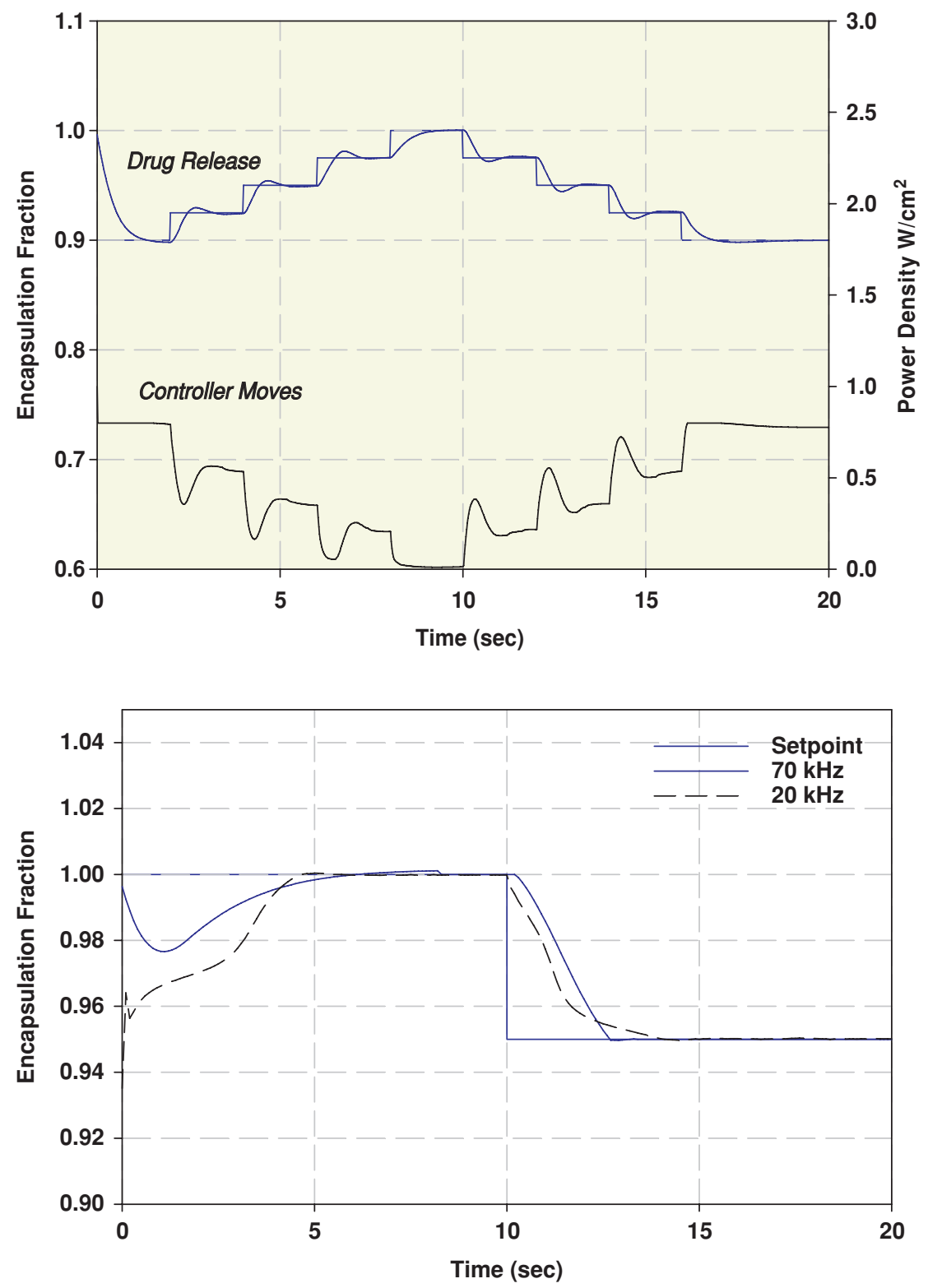

Figure 9: NN-MPC setpoint tracking performance and the corresponding controller (at $70 \mathrm{kHz}$ ).
Figure 10: Step performance of the NN-MPC at 20 and $70 \mathrm{kHz}$. The settling time for $20 \mathrm{kHz}$ and $70 \mathrm{kHz}$ are $4.8 \mathrm{sec}$ and $3.2 \mathrm{sec}$, respectively. The figure shows the response attributed to maintain a 5\% release (or $95 \%$ Dox-encapsulation).
0.95 , or $95 \%$ ). The figure shows the step performance of the NN-MPC at 20 and $70 \mathrm{kHz}$. The settling time for $20 \mathrm{kHz}$ and $70 \mathrm{kHz}$ are 4.8 seconds and 3.2 seconds, respectively. Such step increase in release could be used to maintain constant steady state drug release in vitro and possibly in vivo.

\section{Conclusion}

We have successfully shown that ANN can be used to capture the highly nonlinear dynamics of acoustically activated drug release from polymeric micelles. The developed blackbox model for the drug release process attained high prediction capabilities for all ranges of experimental data. The feed-forward with tapped time delays neural-based model was then utilized to design a nonlinear NN-MPC controller in a model based strategy. The designed controller was effective in controlling the release of Doxorubicin from unstabilized micelles at different frequencies, power intensities and acoustic pulses. However, at $20 \mathrm{kHz}$, more rapid controller effort was needed to guide the drug release to reach its set point. This is mainly due to the process high nonlinearity at this frequency. The modeling and control strategy presented in this paper can be adopted for similar processes where conventional modeling techniques are inefficient due to the highly nonlinear behavior of the process studied.

\section{References}

1. Husseini, G. A., El-Fayoumi, R. I., O’Neill, K. L., Rapoport, N. Y., Pitt, W. G. DNA damage induced by micellar-delivered doxorubicin and ultrasound: comet assay study. Cancer Letters 154, 211-216 (2000). 
2. Husseini, G. A., Rapoport, N. Y., Christensen, D. A., Pruitt, J. D., Pitt, W. G. Kinetics of ultrasonic release of doxorubicin from Pluronic P105 micelles. Coll Surf B: Biointerfaces 24, 253-264 (2002).

3. Husseini, G. A., Myrup, G. D., Pitt, W. G., Christensen, D. A., Rapoport, N. Y. Factors Affecting Acoustically-Triggered Release of Drugs from Polymeric Micelles. J Controlled Release 69, 43-52 (2000).

4. Marin, A., Sun, H., Husseini, G. A., Pitt, W. G., Christensen, D. A., Rapoport, N. Y. Drug delivery in pluronic micelles: effect of highfrequency ultrasound on drug release from micelles and intracellular uptake. J Controlled Rel 84 (1), 39-47 (2002).

5. Husseini, G. A., Runyan, C. M., Pitt, W. G. Investigating the mechanism of acoustically activated uptake of drugs from Pluronic micelles. BMC Cancer 2, (2002)

6. Muniruzzaman, M. D., Marin, A., Luo, Y., Prestwich, G. D., Pitt, W. G., Husseini, G., Rapoport, N. Y. Intracellular uptake of Pluronic copolymer: effects of the aggregation state. Colloids and Surfaces B: Biointerfaces 25(3), 233-241 (2002).

7. Husseini, G. A., Diaz, M. A., Richardson, E. S., Christensen, D. A., Pitt, W. G. The Role of Cavitation in Acoustically Activated Drug Delivery. J Controlled Release 107(2), 253-261 (2005).

8. Husseini, G. A., Diaz de la Rosa, M. A., Gabuji, T., Zeng, Y., Christensen, D. A., Pitt, W. G. Release of Doxorubicin from Unstabilized and Stabilized Micelles Under the Action of Ultrasound. J Nanosci Nanotech 7(3), 1-6 (2007).

9. Husseini, G. A., O’Neill, K. L., Pitt, W. G. The comet assay to determine the mode of cell death for the ultrasonic delivery of doxorubicin to human leukemia (HL-60 cells) from pluronic P105 micelles. Technology in Cancer Research \& Treatment 4(6), 707-711 (2005).

10. Husseini, G. A., Abdel-Jabbar, N. M., Mjalli, F. S., Pitt, W. G. Modeling and sensitivity analysis of acoustic release of Doxorubicin from unstabilized pluronic P105 using an Artificial Neural Network model. Technology in Cancer Research \& Treatment 6 (1), 49-56 (2007).

11. Stevenson-Abouelnasr, D., Husseini, G. A., Pitt, W. G. Further investigation of the mechanism of Doxorubicin release from P105 micelles using kinetic models. Colloids and Surfaces B-Biointerfaces 55(1), 59-66 (2007)

12. Staples, B. J., Pitt, W. G., Schaalje, B., Roeder, B. L. In UltrasonicallyAssisted Drug Delivery in Rats Reduces Tumor Growth, Controlled Release Society, Long Beach, CA, July 7-11, 2007, 2007; Controlled Release Society: Long Beach, CA, 2007; p 839.

13. Stringham, S. B., Viskovska, M. A., Richardson, E. S., Ohmine, S., Husseini, G. A., Murray, B. K., Pitt, W. G. Over-Pressure Suppresses Ultrasonic-Induced Drug Uptake. Ultrasound in Medicine and Biology 35(3), 409-415 (2009).

14. Soma, C. E., Dubernet, C., Bentolila, D., Benita, S., Couvreur, P. Reversion of multidrug resistance by co-encapsulation of doxorubicin and cyclosporin A in polyalkylcyanoacrylate nanoparticles. Biomaterials 21, 1-7 (2000).

15. Kawakami, H., Polymeric membrane materials for artificial organs. Journal of Artificial Organs 11(4), 177-181 (2008).

16. Rapoport, N., Caldwell, K. Structural transitions in micellar solutions of Pluronic P-105 and their effect on the conformation of dissolved Cytochrome $\mathrm{C}$ : an electron paramagnetic resonance investigation. Colloids and Surfaces B: Biointerfaces 3, 217-228 (1994).

17. Yuan, F., Leuning, M., Huang, S. K., Berk, D. A., Papahadjopulos, D., Jain, R. K. Microvascular permeability and interstitial penetration of sterically-stabilized (stealth) liposomes in human tumor xenograft. Cancer Res 54, 3352-3356 (1994).

18. Rapoport, N. Y., Marin, A. P., Timoshin, A. A. Effect of a Polymeric Surfactant on Electron Transport in HL-60 Cells. Arch Biochem Biophys 384(1), 100-108 (2000).

19. Rapoport, N. Y., Herron, J. N., Pitt, W. G., Pitina, L. Micellar delivery of doxorubicin and its paramagnetic analog, ruboxyl, to HL-60 cells: effect of micelle structure and ultrasound on the intracellular drug uptake. J Controlled Rel 58(2), 153-162 (1999).
20. Uhrich, K. E., Cannizzaro, S. M., Langer, R. S., Shakesheff, K. M. Polymeric Systems for Controlled Drug Release. Chem Rev 99, 31813198 (1999).

21. Husseini, G. A., Pitt, W. G. Ultrasonic-Activated Micellar Drug Delivery for Cancer Treatment. Journal of Pharmaceutical Sciences 98 (3), 795-811(2009).

22. Husseini, G. A., Pitt, W. G. Micelles and nanoparticles for ultrasonic drug and gene delivery. Advanced Drug Delivery Reviews 60(10), 1137-1152 (2008).

23. Husseini, G. A., Pitt, W. G. The use of ultrasound and micelles in cancer treatment. Journal of Nanoscience and Nanotechnology 8(5), 2205-2215 (2008).

24. Saxena, J., Sharma, N., Makiod, M. C., Banakar, U. V. Ultrasonically Mediated Drug Delivery. J Biomat Appl 7, 227-296 (1993).

25. Mitragotri, S., Blankschtein, D., Langer, R. Transdermal drug delivery using low-frequency sonophoresis. Pharmaceut Res 13(3), 411420 (1996).

26. Kassan, D. G., Lynch, A. M., Stiller, M. J. Physical Enhancement of Dermatologic Drug Delivery - Iontophoresis and phonophoresis. $J$ Amer Acad Dermatology 34(4), 657-666 (1996).

27. Mitragotri, S., Blankschtein, D., Langer, R. Ultrasound-Mediated Transdermal Protein Delivery. Science 269, 850-853 (1995).

28. Kruskal, J., Goldberg, S., Kane, R. In Novel in Vivo Use of Conventional Ultrasound to Guide and Enhance Molecular Delivery and Uptake into Solid Tumors, Annual Meeting of the Radiological Society of North America, Chicago, IL, Nov 25-30, 2001, 2001; RSNA: Chicago, IL, 2001; p 804.

29. Maeda, $\mathrm{H}$. The enhanced permeability and retention (EPR) effect in tumor vasculature: the keyrole of tumor-selective macromolecular drug targeting. Adv Enzyme Regul 41, 189-207 (2001).

30. Gabizon, A., Catane, R., Uziely, B., Kaufman, B., Safra, T., Cohen, R., Martin, F., Huang, A., Barenholz, Y. Prolonged Circulation Time and Enhanced Accumulation in Malignant Exudates of Doxorubicin Encapsulated in Polyethylene-glycol Coated Liposomes. Cancer Res 54, 987-992 (1994).

31. Kwok, C. S., Mourad, P. D., Crum, L. A., Ratner, B. D. Self-assembled molecular structures as ultrasonically-responsive barrier membranes for pulsatile drug delivery. J Biomed Mater Res 57, 151-164 (2001).

32. Takada, E., Sunagawa, M., Ohdaira, E., Ido, M. Ultrasonic effects on anti-cancer drugs. Ultrasound Med Biol 23(Suppl 1), S132 (1997).

33. Tata, D. B., Biglow, J., Wu, J. R., Tritton, T. R., Dunn, F. Ultrasoundenhanced hydroxyl radical production from two clinically employed anticancer drugs, adriamicin and mitomicin C. Ultrasonics Sonochem 3(1), 39-45 (1996).

34. Saad, A. H., Hahn, G. M. Ultrasound Enhanced Drug Toxicity on Chinese Hamster Ovary Cells in Vitro. Cancer Res 49, 5931-5934 (1989).

35. Bhat, N., McAvoy, T. J. Use of Neural Nets for Dynamic Modeling and Control of Chemical Process Systems. Computers \& Chemical Engineering 14(4-5), 573-583 (1990).

36. Mjalli, F. S., Al-Asheh, S. Neural-networks-based feedback linearization versus model predictive control of continuous alcoholic fermentation process. Chemical Engineering \& Technology 28(10), 1191-1200 (2005).

37. Mjalli, F. S. Neural network model-based predictive control of liquidliquid extraction contactors. Chemical Engineering Science 60(1), 239-253 (2005).

38. Al Seyab, R. K., Cao, Y. Nonlinear system identification for predictive control using continuous time recurrent neural networks and automatic differentiation. Journal of Process Control 18(6), 568-581 (2008).

39. Liu, M. Q., Wang, H. F. A novel stabilizing control for neural nonlinear systems with time delays by state and dynamic output feedback. International Journal of Control Automation and Systems 6(1), 24-34 (2008). 
40. Perez, J. A., Ocana, J. L., Molpeceres, C., Morales, M., Blasco, M. Adaptive neural network control system for laser surface heat treatments. International Journal of Advanced Manufacturing Technology 41(5-6), 513-518 (2009).

41. Etxebarria, V., Adaptive-Control of Discrete-Systems Using Neural Networks. Iee Proceedings-Control Theory and Applications 141(4), 209-215 (1994).

42. Kosanovich, K. A., Piovoso, M. J., Rokhlenko, V., Guez, A. Nonlinear Adaptive-Control with Parameter-Estimation of a Cstr. Journal of Process Control 5(3), 137-148 (1995).

43. Haley, P., Soloway, D. Generalized predictive control for active flutter suppression. Ieee Control Systems Magazine 17(4), 64-70 (1997).

44. Norgaard, M., Ravn, O., Poulsen, N. K., Hansen, L. K. Neural Networks for Modeling and Control of Dynamic Systems. Springer-Verlag: London, 2000.

45. Henson, M. A. Nonlinear model predictive control: current status and future directions. Computers \& Chemical Engineering 23(2), 187 202 (1998).
46. Kurtz, M. J., Henson, M. A. Feedback linearizing control of discretetime nonlinear systems with input constraints. International Journal of Control 70(4), 603-616 (1998).

47. Rapoport, N., Munshi, N., Pitina, L., Pitt, W. G. Pluronic Micelles as Vehicles for Tumor-Specific Delivery of Two Anti-Cancer Drugs to HL-60 Cells Using Acoustic Activation. Polymer Preprints 38(2), 620-621 (1997)

48. Singh-Joy, S. D., McLain, V. C. Safety Assessment of Poloxamers $101,105,108,122,123,124,181,182,183,184,185,188,212,215$, 217, 231, 234, 235, 237, 238, 282, 284, 288, 331, 333, 334, 335, 338, 401, 402, 403, and 407, Poloxamer 105 Benzoate, and Poloxamer 182 Dibenzoate as Used in Cosmetics. International Journal of Toxicology 27, 93-128 (2008)

49. Gilman, A. G., Hardman, J. G., Limbird, L. E., Molinoff, P. B., Ruddon, R. W., Goodman and Gilman's the Pharmacological Basis of Therapeutics. 9th ed.; McGraw-Hill: New York, 1996.

50. Liu, G. P., Kadirkamanathan, V., Billings, S. A. Predictive control for non-linear systems using neural networks. International Journal of Control 71(6), 1119-1132 (1998).

Received: April 2, 2009; Revised: September 1, 2009; Accepted: September 17, 2009 